correction. It is there stated that the patient " spent the last two winters at Mentone, where she was leeched and cauterised fifty times." The original notes are as follows:- "Jan. 21st, 1864: Generally better in chest. Only occasional colds. Of late years, leucorrhœa and uterine irritation sometimes relieved by leeches before period. Last two winters at Mentone; had canstic fifty times, and better, but still cannot walk much. Taken oil, \&c." It appears, therefore, that there was no leeching at Mentone; and the patient's own expression, that the caustic was applied fifty times, was probably an exaggeration, and merely meant to signify a great number of times.

( $T o$ be continued.)

\section{THE INOCULATION OF TUBERCLE IN ITS RELATION TO CLINICAL EXPERIENCE.}

Bx SAMUEL WILKS, M.D. LoND., PHYSICLAN TO GUY'S HOSPITAI.

Tres subject of the inoculation of tubercle, originated by Villemin and other foreign experimenters, and which is now undergoing a rigid examination at the hands of such excellent observers as Mr. Simon, Dr. Andrew Clark, Dr. Sanderson, and Dr. Wilson Fox, must be treated entirely on its own merits, and should in no way be prejudiced by the introduction into it of extraneous matter. The investigations by these gentlemen may serve as models for the manner in which such inquiries should be undertaken, and we may hope that they may be pushed still further until some definite conclusion is arrived at. It is quite impossible, however, to prevent the medical mind from speculating at once upon their nature, and making those applications of the facts which these at present seem to warrant. Thus already the announcement of the inoculability of tubercle has been sufficient to induce some in the profession to assert their belief in the accidental character of the disease known as consumption. For my own part, I think the facts are at present too crude to allow of any deductions which can be applicable to the elucidation of the question of tuberculosis in the human subject; much less can they be taken as exemplifying a universal law of the propagation of tubercle in the animal kingdom. Since, however, there are those who have accepted the results already obtained as affording an explanation of the ravages of tuberculous disease in mankind, I will briefly state that at present our clinical experience presents most formidable objections to the reception of the new doctrine.

The first announcement, it may be remembered, was simply that tubercle could be inoculated. This being apparently subversive of the common opinion that tuberculosis was a constitutional disease, the further declaration that the same results were attainable by the use of many animal substances was received with much satisfaction, for it tended to the conclusion that the effect produced was of the nature of pyæmia. Subsequently, however, the elaborate researches of Dr.Wilson Fox and others proved that the new formations are real growths of cell composition, and that truly they can be produced by the inoculation of a variety of substances into the system. The latter, however, are more or less allied, being what are styled homologous elements-that is, cell formations, the result of inflammatory processes in different tissues of the body. I am not aware that any material supposed to contain any specific elements in its constitution has been found equally effective. Both Dr. Sanderson and Dr. Wilson Fox, I believe, in contemplating their experiments, see no difficulty in regarding all these new growths, which they have been instrumental in making, as identical in composition, and thus give to them the appellation of tubercle; and they then, moreover, naturally divert to the domain of human pathology, and inquire whether we have not been too stringent in the definition of this deposit, and been making distinctions where none existed ; whether, indeed, the crude or cheesy deposits so constantly found in the human subject should not all be regarded as tubercle.

There need be no fear of such cautious as well as intelligent observers rushing into theoretical speculations whose truth their experience might not confirm ; but yet there are those persons who would at once draw an inference to the effect that all the amorphous and caseous deposits found in the human body deserve the name of tubercle, and that they may originate as it were by accident. Of course there is no difficulty in so wide-spread form of disease for some persons, with whom I have conversed on the subject, to bring forward instances of phthisis succeeding to a chronic synovitis, an abscess in the testis, or a fistula in ano, to say nothing of the many examples of a husband soun following a wife to the grave, or a sister the brother, in confirmation of the opinion that the seeds of tubercle may be carried by the breath. Now, although it is no difficult task to select instances of this nature which may tend to corroborate such a doctrine as that just mentioned, yet we must look up our whole clinical experience in order to discover how far it can be applied; and in doing so I cannot but think that this will be found quite out of harmony with the teaching which the experiments might seem to warrant. The subject is a very large one, and cannot be treated in a few words; it involves the whole question of what is understood by tubercle, what is meant by local and constitutional disease, and how the term phthisis is understood by the profession.

If it should be said that the material which is found in a dried-up abscess or lymphatic gland which has been the subject of inflammation is the same material as that discovered in the body in a case of consumption, I would answer, that although the naked eye may not perceive a distinction, yet they are to all intents and purposes different. It might, however, be said again that this is assuming the very point to be determined, which is, whether any material accidentally produced on the surface, as from injury, may not be propagated through the system in a form which is usually styled tuberculous, and which all admit tends only to one event. I would say a secend time that this is the arena for the combat of the rival doctrines, and that clinical observation is altogether opposed to the idea of the spread of tubercle in this accidental manner; in fact, I myself know of no form of disease to which the doctrine apparently arising from the experiments mentioned would less apply than that of tuberculosis; it would much more fit the facts observed concerning the growth of cancer.

The question concerning the local and constitutional nature of any disease is as old as it is important. The tendency of all clinical experience has been to favour the constitutional rather than the accidental cause of disease; whilst pure pathology has had much to advance on the other side. Thus I have on various occasions attempted to show that particular tissues of the body are liable to special changes, whilst they are at the same time insusceptible to others; that, for example, it is not true, as was once supposed, that a cancer, a tubercle, or an abscess can spring up de novo in any part of the body. I believe that a cancer or an abscess in the lung is one of the rarest possible occurrences as an idiopathic affection, and that almost invariably it may be shown that the seeds of the disease have been brought from a distant part. In the case of cancer, although one cannot deny the hereditary disposition to the disease, ye my own observations, like those of Mr. Moore, tend to prove its frequent local origin. Indeed, the example of osteoid cancer, originating in bone, and melanotic cancer, often having its source on a pigmental mole, is sufficient to show how local characters of a disease are propagated. I might also mention the occasional case of epithelial elements being discovered in solid organs which neighbour on an epithelial growth. When, therefore, it is said that abnormal deposits on the surface tend to the formation of similar deposits in the interior of the body, we have clinical experience of the fact. Even if cancer, in its various compound forms, had been inoculated in animals, I believe we could have advanced nothing in human pathology in opposition to the experiments. If a local formation of a peculiar character is succeeded by similar formations in the organs of the body, whether these are composed of nuclei or cells of cancer-the cells of pus, bonetissue, or pigment, -we are not surprised at those ill-formed cell-structures which are found in inflamed products being succeeded by like growths in the interior of the body, if the former be inoculated. This has yet to be proved as occurring with frequency in the human body, although I think it possible, as one or two remarkable instances coming under my own notice have shown, in which the question arose between a pyæmic process or a case of multiple growths. But, admitting these facts, what concerns us here is the question whether these inoculable deposits are deserving of the name "tubercle." My own opinion is that they are not; indeed, I confess to the fact that all my teaching of late years has been towards the overthrow of the opinion that all low-organised or caseous deposits are to be regarded as tuberculous, seeing that they arise under such a variety of circumstances, which preclude their having anything in common. 
The most important objection against the inoculability of tubercle and the accidental nature of tuberculosis being allowed a status in the human subject is, that there is very little evidence clinically of tubercle having any origin which would corroborate such views; whilst, on the other hand, in the great majority of instances it apparently springs up spontaneously. Indeed, if there is any one fact in medicine which has a firm foundation it is the hereditary disposition to consumption. It is indeed so certain, that if the inoculability of tuberc'e in the human subject were proved, it would be equivalent to the fact of small-pox being a specific disease, and yet only capable of attacking certain individuals-a fact which would practically be changing its nature from a specific to a constitutional affection. I think the hereditary tendency to disease is one of the most important facts for the consideration of medical men, and in no case more so than that of tuberculosis. The type of the tuberculous subject is so well marked that the young man or maiden possessing it may be picked out long before any manifestation of disease has displayed itself. All the members of a family may be seen to fall into the grave one after another, whilst the same circumstances which surrounded them have no influence on those who may be dwelling in the adjoining mansion. Of all the practical objections against the idea of the accidental propagation of tubercle, this is the most important. It is one which, to my mind, entirely precludes the idea of consumption, in the large majority of instances, having any such origin; and, as regards treatment, the recognition of the diathesis, or the inclination towards consumption, is, I consider, more important than any amount of knowledge respecting the actual existence of the disease in the body. This hereditary tendency appears to me so fully ascertained, that even if the inoculability of tubercle were proved to be possible in the human subject, $I$ believe it would bear very little on the actual production of the disease as at present seen in consumptive persons.

Moreover, the development of tubercle in the body does not follow the same rule as do those forms of deposit which are propagated from without-as, for instance, in pyæmia or cancer, which, commencing on the surface, subsequently involve the lungs and other parts. In both these forms of disease there is frequently a history of an injury; and in the case of cancer, when situated externally, the surgeon does not hesitate to remove it for fear of similar disease within, for this he knows occurs at a later period. But how rarely does tuberculosis possess a history of this kind. In infants the brain is more susceptible to the development of tubercle; in children, the abdomen; in adults, the lungs. In fatal phthisis there is found, in the majority of instances, tubercles in the lungs and the intestines; but nowhere else. These examples prove, I believe, that there are especial circumstances within the body which determine the location of the disease, the peculiarities of which are not readily accounted for on the supposition of the introduction of tubercle from without. Then there is the negative fact that no such cause as this is apparent in the majority of cases; and also the other negative fact, that local disease may remain for years without the body generally being implicated in it. A suppurating lymphatic gland or an abscess in the testis may exist for years without being followed by any absorption of inflammatory material; and certainly the cases of diseased joints which are not attended by phthisis form a majority over those where the two diseases are associated. Surgeons have been hitherto remiss in not offering to the profession fuller statistics as to the frequency of the association of the two. Everyone must have seen an apparent phthisis arrested by removal of a limb, as well as many cases where phthisis has succeeded to the operation. Then, again, I might mention such a purely local disease as that found in morbus Addisonii, and where the material in the suprarenal capsule appears identical with that of tuberculous matter-a disease so local as to preclude its constitutional origin, at the same time not admitting the app.ication of a newer doctrine. The cases of phthisis in connexion with fistula in ano should be more closely watched, in order to make the discovery, if possible, that the pulmonary disease has succeeded to an abscess in the rectum which has been set up purely by accidental circumstances. One cannot but think that, if a chronic inflammatory process on the surface of the body was liable to produce a general disease through the organs, the fact would have been long ago observed, and that a disease like phthisis rould have been as much connected with surgical operations as the acuter affection pyiemia now is.

The different circumstances under which deposits very much alike in their character may occur in the body would show that they can have very little in common which can be of any ralue in a pathological or therapeutical point of view. Supposing that, owing to the absorption of some material, a disease was set up in the lungs which in ordinary parlance was styled phthisis; and supposing that a syphilitic or diabetic patient had a low form of pneumonia in which caseous matter was formed, and a similar disorganisation followed, such cases would have very little resemblance except in their final stages; for all practical purposes they would be different. The first and most important objections, then, against a disease like phthisis being produced from without, and therefore liable to be set up by accidental causes, are, the well-known facts of its hereditary nature, and the absence in the majority of cases of any such exciting causes as are indicated; and, on the contrary, the negative fact of the thousands of patients who might be found daily with external wounds, without any im. plication of internal parts. Then as regards the mode in which tubercle attacks the human body, which is so unlike disease which has been introduced after the manner of pyæemia or secondary growths. I would, however, allow that there may be varieties of the so-called tuberculous disease, as well as different modes of propagation, for everyone of any experience must have observed how, in some cases of scrofulous or tuberculous disease, very different tissues are attacked. Thus, in one case the whole of the solid organs are found stuffed with tubercles; in another case these are quite free, but the serous membranes are covered with the deposits; whilst in a third the lymphatic glands are the parts involved. I am here reminded that the production of tubercle resulting from the inoculation in animals would tend to subvert the opinion which holds to the difference between the scrofulous and the tuberculous diatheses, an opinion which I strongly maintain.

The question, by way of illustration, might be put thus : What is there in common between a case of ordinary consump. tion, one of morbus Addisonii, and one of chronic pulmonary disease succeeding to diabetes or syphilis? In all of these a cheesy matter may be found in some organ of the body, to which the name "scrofulous" or "tuberculous" is usually given. Is this a link sufficiently important to bind them by a common name?-or are not consumption, diabetes, syphilis, and morbus Addisonii, constitutionally, pathologically, and therapeutically distinct?

The subject thus briefly referred to is one of very great importance, and the discussion upon it might be protracted to any length ; and therefore I will only say, in conclusion, that these remarks are not intended to throw any doubt upon the experiments performed by the distinguished observers before named-for no one has felt more interest in those experiments than myself,-but they are rather intended for such persons who pass through a medical life without having obtained any fixed opinions respecting the nature and treatment of disease, and who are ready to exclaim, on the publication of any novel theory or experiment, that the whole of one's former experience is to be subverted. The experimenters themselves have not yet applied their observations to human disease, although others may have done so for them. I trust that they will continue their observations, since the results already obtained open up a large field of inquiry, and in fact, from many points of view, have reference to some of the most important questions in pathology.

St. Thomas'street, S.F., Alay, 1868.

NOTE ON THE

\section{ARTIFICIAL PRODUCTION OF TUBERCLE.}

BY RICHARD PAYNE COTTON, M.D., SENTOR PHYSICIAN TO THE HOSPITAX FOR CONSUMPTION, BBOMPTON.

THE striking experiments of M. Villemin (Bull. Acad. Méd., xxxi., xxxii.) on the inoculation of tubercle, appearing as they did at first to justify at least the grave suspicion that, in spite of all that might be said to the contrary, phthisis was a contagious disease, have happily been negatived by more recent investigations. I use the word happily, because I know of nothing more depressing than the belief that consumption-a disease which demands for a lengthened period, not only sympathy, but the closest personal devotion-is capable of being communicated by the ordinary laws of contagion.

Dr. William Budd, in a recent paper in The LANCET (Oct. 12 th, 1867), fortified as it might seem by M. Villemin's statements, somewhat startled the profession by boldly declaring 